\title{
Estimación de la exposición a fluoruros en Los Altos de J alisco, México
}

\author{
Roberto Hurtado-Jiménez, M en $C_{1}^{(1,2)}$ Jorge $G$ ardea-Torresdey, PhD. ${ }^{(2,3)}$
}

\section{Hurtado-Jiménez R, Gardea-Torresdey J. Estimación de la exposición a fluoruros en LosAltos de Jalisco, México.} Salud Publica Mex 2005;47:58-63. El texto completo en inglés de este artículo está disponible en: http://www.insp. mx/salud/47/eng

\section{Resumen}

Objetivo. Estimar la exposición a fluoruros y riesgos potenciales a la salud humana en Los Altos de Jalisco, México. Material y métodos. Se midió electroquímicamente la concentración de fluoruros en 105 pozos y seis tomas de agua potable, en losA Itos de Jalisco, de mayo a julio de 2002. Se estimó la dosis de exposición y la ingestión total de fluoruros para: bebés de $10 \mathrm{~kg}$, niños de $20 \mathrm{~kg}$ y adultos de $70 \mathrm{~kg}$. Resultados La concentración de fluoruros en las muestras de agua varió entre 0.1 y $17.7 \mathrm{mg} / \mathrm{l}$. El $45 \%$ de las muestras excede el límite permitido por la normatividad $(1.5 \mathrm{mg} / \mathrm{l})$. La ingestión total y dosis de exposición a fluoruros estimados están en los rangos de 0.5-18.4 mg/d y 0.04-1.8 mg/kg/d, respectivamente. Conclusiones. Una parte importante de la población está expuesta a fluorosis dental, fluo rosis esquelética y fracturas óseas. Para reducir los riesgos se debe evitar el consumo de sal fluorada, pastas dentales con flúor y agua potable cuya concentración de fluoruros sea mayor de $0.7 \mathrm{mg} / \mathrm{l}$. El texto completo en inglés de este artículo está disponible en:http://www.insp.mx/ salud/47/eng

Palabras clave: flúor; fluo ruros; ingestión de fluoruros; fluorosis; México

\author{
Hurtado-Jiménez R, Gardea-Torresdey J. \\ Estimation of exposure to fluoride \\ in "LosAltos de Jalisco", México. \\ Salud Publica Mex 2005;47:58-63. \\ The English version of this paper \\ is available at: http://www.insp.mx/salud/47/eng
}

\begin{abstract}
A bstract
Objective.To estimate the level of fluoride exposure and human health risks in Los Altos de Jalisco (Jalisco State Heights) region. Material and Methods This study was conducted between May and July 2002. The fluoride concentrations of 105 water wells and six tap water samples were electrochemically measured. Exposure doses to fluoride and total intake of fluoride were estimated for babies $(10 \mathrm{~kg})$, children $(20 \mathrm{~kg})$, and adults $(70 \mathrm{~kg})$. Results. The fluoride concentration of the water samples ranged from 0.1 to $17.7 \mathrm{mg} / \mathrm{l}$. More than $45 \%$ of the water samples exceeded the national guideline value for fluoride of 1.5 $\mathrm{mg} / \mathrm{l}$.The estimated values of the exposure doses to fluoride and total intake of fluoride were in the range of 0.04-1.8 $\mathrm{mg} / \mathrm{kg} / \mathrm{d}$ and $0.5-18.4 \mathrm{mg} / \mathrm{d}$, respectively. Conclusions D ental fluo rosis, skeletal fluo rosis, and bone fractures are so me of the potential health risks due to the intake of high doses of fluoride for the population of Los Altos de Jalisco. In order to reduce health risks, fluo ridated salt, fluoridated to othpastes, and drinking water containing more than $0.7 \mathrm{mg} / \mathrm{l}$ of fluoride should be avoided. The English version of this paper is available at: http://www.insp.mx/salud/47/eng
\end{abstract}

Key words: fluorine; fluorides; fluoride intake; fluorosis; Mexico

(1) El Colegio de la Frontera N orte,A.C. Ciudad Juárez, Chihuahua, México.

(2) Programa de Doctorado en Ciencias e Ingeniería A mbiental, Universidad de Texas en el Paso, Estados Unidos de América.

(3) Departamento de Q uímica, U niversidad de Texas, Estados U nidos de A mérica.

Fecha de recibido: 26 de agosto de 2004 • Fecha de aprobado: 15 de diciembre de 2004

Solicitud de sobretiros: Maestro Roberto Hurtado Jiménez,Avenida Insurgentes N 0. 3708

Fraccionamiento Los N ogales, Ciudad Juárez 32350, C hihuahua, México.

Correo electrónico: rhurtado@ colef.mx 
La región conocida como Los Altos de Jalisco (LAJ), con una población de 696318 habitantes $^{1}$ asentados en 20 municipios, se encuentra ubicada en la parte nordeste del estado de Jalisco. Esta región forma parte del Cinturón Volcánico Trans-Mexicano, caracterizado por la termalidad de las aguas subterráneas. ${ }^{2}$

La fuente principal de suministro de agua potable, en la mayoría de las poblaciones de LAJ, es agua subterránea, que en muchos casos es de origen hidrotermal. Las aguas termales se caracterizan por la presencia de elementos químicos potencialmente tóxicos. Uno de estos elementos es el flúor. La concentración máxima de fluoruros $\left(\mathrm{F}^{-}\right)$en el agua potable, que permite la normatividad mexicana, ${ }^{3}$ es de $1.5 \mathrm{mg} / \mathrm{l}$.

La fuentes principales de exposición a $\mathrm{F}^{-}$en LAJ, además del agua, son sal fluorada, pastas dentales, algunas bebidas y jugos embotellados, y algunas variedades de té y café.

Las principales enfermedades ocasionadas por la ingestión prolongada de altas dosis de $\mathrm{F}^{-}$incluyen: fluorosis dental ${ }_{1}^{4-6}$ fluorosis esquelética, ${ }^{6,7}$ deformación de huesos ${ }^{6,7}$ fracturas (principalmente de cadera) ${ }_{,}^{8}$ diversos tipos de cáncer ${ }^{9}$ y envejecimiento prematuro. ${ }^{9}$

Las principales ciudades mexicanas donde el agua potable tiene un exceso de $\mathrm{F}^{-}$son: Aguascalientes, ${ }^{10}$ Chihuahua, ${ }^{11}$ Durango, ${ }^{8}$ Hermosillo, ${ }^{12}$ Salamanca ${ }^{13}$ y San Luis Potosí, ${ }^{5,13-16}$

Los objetivos del presente trabajo son: a) identificar las poblaciones de LAJ donde la concentración de fluoruros ([ $\left.\mathrm{F}^{-}\right]$) en el agua potable excede los límites permitidos; b) estimar la ingestión diaria y dosis de exposición a $\mathrm{F}^{-}$de los habitantes de LAJ, incluyendo otras fuentes, y (c) identificar las enfermedades fluoróticas que podrían prevalecer en LAJ en función de la exposición al flúor.

\section{Material y métodos}

Se tomaron 105 muestras de agua de pozos en operación y seis muestras de agua de la llave en 18 cabeceras municipales de LAJ. El muestreo se realizó de mayo a julio de 2002 siguiendo los procedimientos convencionales. No se hizo ningún tratamiento especial a las muestras, dada la estabilidad de $\mathrm{F}^{-}$en soluciones acuosas. En Cuquío y Yahualica de González Gallo se tomaron muestras del agua de la llave, ya que no se utilizan pozos para el suministro de agua.

El flúor se cuantificó utilizando el método para la determinación de $\mathrm{F}^{-}$recomendado por la Agencia de Protección Ambiental de los Estados Unidos (Método 340.2). ${ }^{17}$ La temperatura y el $\mathrm{pH}$ del agua se midieron en el sitio de muestreo.
La ingestión total de $\mathrm{F}^{-}(\mathrm{mg} / \mathrm{d})$ se calculó por medio de la siguiente ecuación:

$I F_{\text {Total }}=I F_{\text {Agua }}+I F_{\text {Sal fluorada }}+I F_{\text {Alimentos }}+I F_{\text {Dentrificos }}+I F_{\text {Bebidas }}$

en la que

$$
I F_{i}=C_{i} V_{i}
$$

donde

$$
\begin{gathered}
C_{i}=\begin{array}{c}
\text { concentración de } \mathrm{F}^{-} \text {en el elemento ingerido, } \\
\mathrm{mg} / \mathrm{ló} \mathrm{mg} / \mathrm{kg}
\end{array} \\
V_{i}=\text { cantidad del elemento ingerido, } 1 / \mathrm{d} \mathrm{o} \mathrm{kg} / \mathrm{d}
\end{gathered}
$$

La dosis de exposición a $\mathrm{F}^{-}, D E_{j}$ se determinó usando la siguiente ecuación:

$$
D E_{j}=\frac{\sum_{i} C_{i} V_{i}}{W_{j}}
$$

donde

$D E_{j}=$ dosis de exposición a $\mathrm{F}^{-}$de la persona $\mathrm{j}, \mathrm{mg} / \mathrm{kg} / \mathrm{d}$

$W_{j}=$ peso de la persona $\mathrm{j}, \mathrm{kg}$

La estimación de la ingestión diaria y la dosis de exposición a $\mathrm{F}^{-}$se hizo para tres casos: caso 1: bebé de $10 \mathrm{~kg}$; caso 2: niño de $20 \mathrm{~kg}$, y caso 3: adulto de 70 $\mathrm{kg}$. Las estimaciones están basadas en la siguiente información: a) el bebé consume diariamente $500 \mathrm{ml}$ de jugos de frutas $\left(0.6 \mathrm{mg} \mathrm{F}^{-} / \mathrm{l}\right)$ y una fórmula alimenticia que requiere de $750 \mathrm{ml}$ de agua de la llave hervida; b) el niño consume diariamente $750 \mathrm{ml}$ de agua embotellada (0.7 $\left.\mathrm{mg} \mathrm{F}^{-} / 1\right), 250 \mathrm{ml}$ de agua hervida, $250 \mathrm{ml}$ de bebidas emvasadas $\left(0.7 \mathrm{mg} \mathrm{F}^{-} / \mathrm{l}\right), 1.9 \mathrm{~g}$ de sal fluorada (250 $\left.\mathrm{mg} \mathrm{F}^{-} / \mathrm{kg}\right)$, pasta de dientes $\left(0.6 \mathrm{mg} \mathrm{F}^{-} / \mathrm{d}\right)$ y una dieta normal de alimentos regionales $\left(0.4 \mathrm{mg} \mathrm{F}^{-} /\right.$ d), y c) el adulto consume $1500 \mathrm{ml}$ de agua embotellada, $350 \mathrm{ml}$ de agua hervida, $350 \mathrm{ml}$ de bebidas envasadas, $6.9 \mathrm{~g}$ de sal fluorada, pasta de dientes y una dieta normal de alimentos regionales. Los valores utilizados en la simulación se establecieron a partir de información existente. ${ }^{12,16,18,19-21}$

La evaluación de la exposición a $\mathrm{F}^{-}$está basada en recomendaciones de varias instituciones científicas, $^{4,21-23}$ las cuales recomiendan los rangos de ingestión de $\mathrm{F}^{-}$para los tres casos considerados: a) bebé: $0.4-2.2 \mathrm{mg} / \mathrm{d}$; b) niño: 0.8-2.4 mg/d; y c) adulto: $2.0-10.0 \mathrm{mg} / \mathrm{d}$. En cualquiera de los casos la dosis de exposición no debe ser mayor de $0.05 \mathrm{mg} / \mathrm{kg} / \mathrm{d}$. 


\section{Resultados}

Los resultados presentados en el cuadro I indican que: a) la $\left[\mathrm{F}^{-}\right]$en el agua de los pozos muestreados varía entre 0.1 y $17.7 \mathrm{mg} / \mathrm{l}$; b) En nueve ciudades la [F-] promedio en el agua de los pozos es mayor de $1.5 \mathrm{mg} / \mathrm{l}$. En ocho de estas ciudades la temperatura promedio del agua es mayor de $30^{\circ} \mathrm{C}$; c) en cuatro ciudades todos los pozos muestreados tienen una $\left[\mathrm{F}^{-}\right]$mayor de $1.5 \mathrm{mg} / \mathrm{l}$, y la temperatura promedio es mayor $\operatorname{de} 30^{\circ} \mathrm{C}$; d) las $\left[\mathrm{F}^{-}\right]$mas altas obtenidas en este muestreo fueron de $17.7 \mathrm{mg} / \mathrm{l}$ en el Pozo No 4 de Teocaltiche, $17.2 \mathrm{mg} / \mathrm{l}$ en el Pozo No. 3 de Mexticacán y 14.9 mg/l en el Pozo No. 10 de Tepatitlán de Morelos, y d) 49\% de los pozos muestreados exceden el límite máximo de $\mathrm{F}^{-}$permitido por la normatividad.

Las estimaciones de ingestión total de $\mathrm{F}^{-}$variaron entre $0.5-18.4,0.8-8.1$, y $4.5-12.7 \mathrm{mg} / \mathrm{d}$ para bebés, niños y personas adultas, respectivamente. Las dosis de exposición a $\mathrm{F}^{-}$fueron de $0.04-1.80,0.11-0.40$, y $0.06-0.18 \mathrm{mg} / \mathrm{kg} / \mathrm{d}$ para bebés, niños y personas adul- tas, respectivamente. Las poblaciones más expuestas son Mexticacán y Teocaltiche donde se rebasan los límites permitidos.

La figura 1 incluye dos gráficas para cada uno de los tres casos seleccionados. En la primera serie se muestra la ingestión de $\mathrm{F}^{-}(\mathrm{mg} / \mathrm{d})$ para los cinco tipos de aguas, según la clasificación de Hurtado y GardeaTorresdey. ${ }^{18}$ Para reducir el número de iteraciones y simplificar los cálculos, las concentraciones de cada tipo de agua corresponden al promedio del rango, esto es $0.4,1.1,2.8$, y $7.0 \mathrm{mg} \mathrm{F}^{-} / 1$ para los tipos A, B, C y D; en el caso del tipo $\mathrm{E}$ se consideró un valor arbitrario de $14.0 \mathrm{mg} \mathrm{F}^{-} / 1$. La otra serie de gráficas muestran en forma comparativa la contribución de $\mathrm{F}^{-}$de cada una de las fuentes.

En general, las gráficas muestran que: a) para los bebés la fuente principal de $\mathrm{F}^{-}$es el agua de la llave; $\mathrm{b}$ ) para los niños los tres factores de riesgo mas importantes son el agua de la llave, la sal de mesa y las pastas dentales, y c) para los adultos la fuentes principales de flúor son la sal de mesa y el agua de la llave.

\section{Cuadro I \\ Concentración promedio de fluoruros en pozos mUestreados en poblaciones de Los Altos de Jalisco, México, 2002}

\begin{tabular}{|c|c|c|c|c|c|}
\hline Cabecera M unicipal & Población & Pozos & $\mathrm{pH}$ & Temperatura ${ }^{\circ} \mathrm{C}$ & Fluoruros* (mg/l) \\
\hline Acatic & 11005 & 4 & 6.9 & 32.8 & $1.7 \pm 1.1(0.2-2.7)$ \\
\hline Arandas & 39478 & 5 & 7.0 & 26.2 & $0.1 \pm 0.0(0.1-0.1)$ \\
\hline Cuquío & 4101 & $(1)^{\ddagger}$ & 5.8 & 23.7 & $0.3 \pm 0.0(0.1-0.1)$ \\
\hline Encarnación de Díaz & 20772 & 5 & 7.3 & 30.9 & $4.5 \pm 1.3(2.5-5.9)$ \\
\hline Jesús María & 7852 & 4 & 7.1 & 27.7 & $0.1 \pm 0.0(0.1-0.1)$ \\
\hline Lagos de Moreno & 79592 & 13 & 7.5 & 34.8 & $3.2 \pm 1.4(1.1-5.2)$ \\
\hline Mexticacán & 3603 & 4 & 7.4 & 37.9 & $6.6 \pm 7.2(0.9-17.2)$ \\
\hline O juelos de Jalisco & 9338 & 3 & 6.9 & 38.7 & $1.9 \pm 1.1(1.3-3.1)$ \\
\hline San Diego de Alejandría & 4749 & 2 & 7.8 & 27.4 & $0.5 \pm 0.0(0.5-0.5)$ \\
\hline San Juan de los Lagos & 42411 & 10 & 7.3 & 32.8 & $4.3 \pm 1.1(2.5-5.9)$ \\
\hline San Julián & 12117 & 2 & 7.1 & 26.2 & $0.4 \pm 0.1(0.3-0.4)$ \\
\hline San Miguel El Alto & 21098 & 5 & 7.2 & 24.6 & $0.6 \pm 0.1(0.5-0.7)$ \\
\hline Teocaltiche & 21518 & 5 & 7.0 & 30.2 & $7.6 \pm 5.9(3.1-17.7)$ \\
\hline Tepatitlán de Morelos & 74262 & 24 & 6.8 & 30.9 & $2.4 \pm 3.8(0.1-14.9)$ \\
\hline Unión de San Antonio & 6317 & 3 & 7.2 & 27.1 & $1.5 \pm 0.8(0.6-2.2)$ \\
\hline Valle de Guadalupe & 4178 & 4 & 7.4 & 35.7 & $3.2 \pm 0.7(2.5-3.9)$ \\
\hline Villa Hidalgo & 11552 & 12 & 6.6 & 28.8 & $0.6 \pm 0.3(0.4-1.3)$ \\
\hline Yahualica de González Gallo & 14225 & $(2)^{\ddagger}$ & 5.8 & 22.0 & $0.3 \pm 0.0(0.3-0.3)$ \\
\hline $\begin{array}{l}\text { Total } \\
\text { * Fluoruros = concentración } \\
\text { Rango de concentraciones } \\
\text { ₹ A gua de la llave }\end{array}$ & $\begin{array}{l}\text { desviación } \\
\text { ción mínim }\end{array}$ & $\begin{array}{l}105 \\
\text { ación } m\end{array}$ & & & $2.5 \pm 3.2(0.1-17.7)$ \\
\hline
\end{tabular}



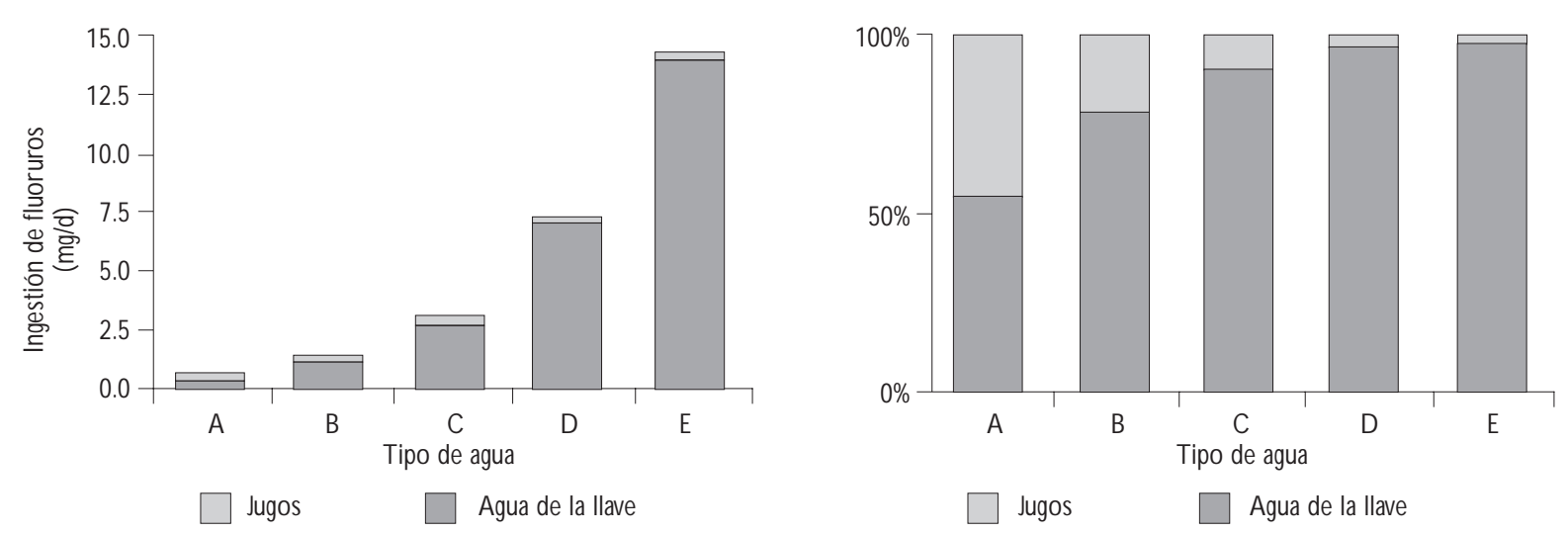

Caso 1. Bebé de 12 meses de edad (10 kg de peso)
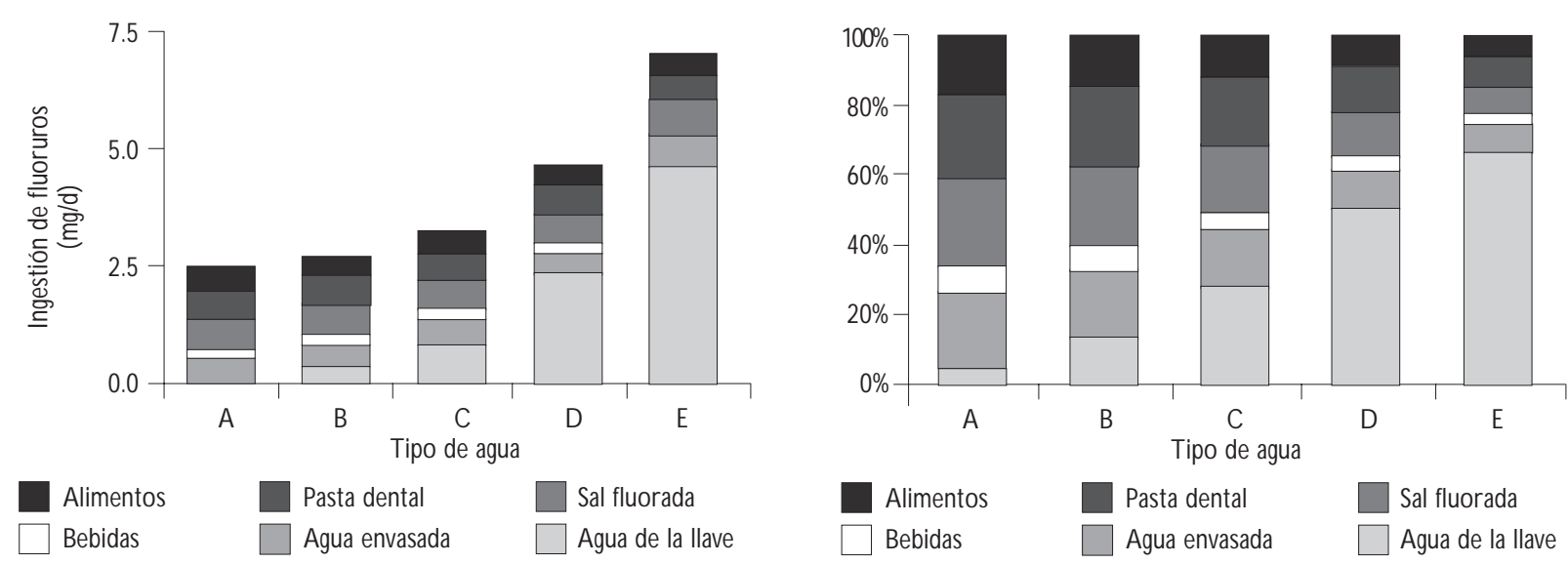

Alimentos

Pasta dental

$\square$ Sal fluorada

Bebidas

A gua envasada

$\square$ Agua de la llave

Agua de la llave

Caso 2. N iño de seis años de edad ( $20 \mathrm{~kg}$ de peso)

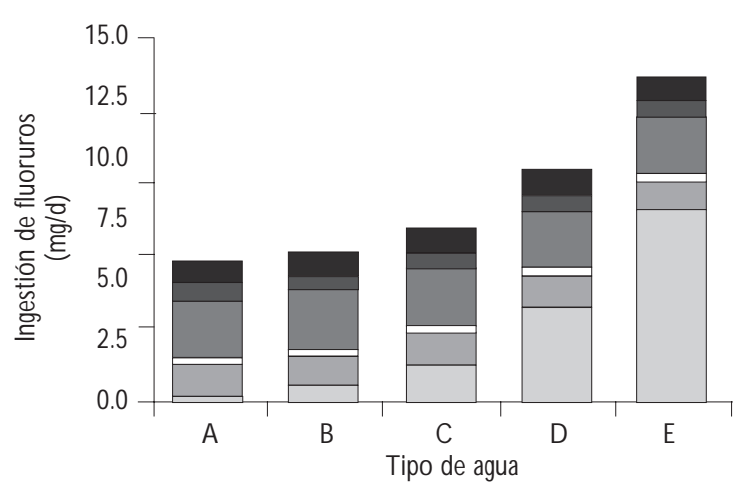

Alimentos Bebidas
Pasta dental

A gua envasada

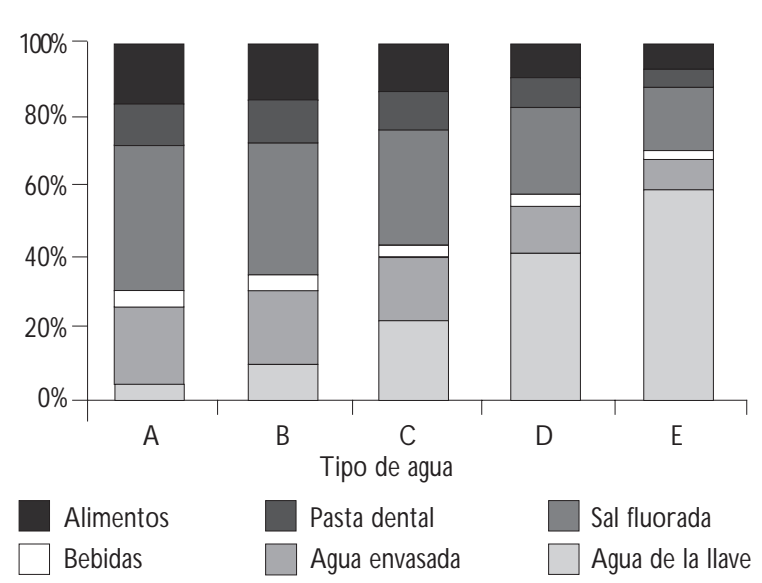

Agua de la llave

Caso 3. Adulto mayor de 25 años de edad (70 kg de peso)

Figura 1. Ingestión total de fluoruros (Mg/D) y su distribución porcentual en ciudades de Los Altos de Jalisco con diferente tipo de agua (clasificación de Hurtado y Gardea-Torresdey). ${ }^{18}$ México, 2002 


\section{Discusión}

El impacto sobre la salud debido a la ingestión prolongada de $\mathrm{F}^{-}$depende de tres factores principales: ingesta diaria de $\mathrm{F}^{-}$, grado de nutrición y status de algunos órganos vitales. La ingesta diaria depende básicamente de la [F-] en el agua potable, consumo de sal fluorada y uso de pasta dental con flúor.

Las enfermedades fluoróticas más comunes son: fluorosis dental, fluorosis esquelética y fracturas óseas. Estudios epidemiológicos efectuados en San Luis Potosí mostraron ${ }^{5}$ una prevalencia de $98 \%$ en comunidades que consumían agua con una $\left[\mathrm{F}^{-}\right]$de $2.0 \mathrm{mg} / \mathrm{l}$. En algunas poblaciones de la India, ${ }^{6}$ donde la $\left[\mathrm{F}^{-}\right]$en el agua varía entre 3.2 y $4.0 \mathrm{mg} / \mathrm{l}$, la prevalencia de fluorosis dental alcanza valores de $77 \%$, mientras que en otras con una concentración similar, la prevalencia fue de $100 \%$, por deficiencias nutricionales.

Los riesgos de ocurrencia de fluorosis dental en LAJ son muy altos, ya que gran parte de la población consume agua potable (embotellada o de la llave) con una $\left[\mathrm{F}^{-}\right]$mayor de $1.5 \mathrm{mg} / \mathrm{l}$. Los valores esperados para LAJ, serían similares a los observados en San Luis Potosí. En México, la fluorosis dental es un mal endémico que afecta a cerca de cinco millones de personas. ${ }^{13}$

La prevalencia de fluorosis esquelética en Rajasthan, ${ }^{6}$ India, fue de $32.8-39 \%$, donde la $\left[\mathrm{F}^{-}\right]$en el agua está entre 3.2 y $4.0 \mathrm{mg} / 1$. En la población hindú de Banda Ganti se encontró una prevalencia de $53 \%$ al consumir agua con 3.8-6.7 $\mathrm{mg} \mathrm{F}^{-} / 1 .{ }^{23}$ Aunque no hay reportes de prevalencia de fluorosis esquelética en LAJ, las poblaciones de Teocaltiche, Mexticacán y Temacapulín (en Cañadas de Obregón) son de alto riesgo ya que la $\left[\mathrm{F}^{-}\right]$ es muy alta. 2,18

Un estudio realizado en la ciudad de Durango, ${ }^{8}$ donde la $\left[\mathrm{F}^{-}\right]$en el agua potable varía entre 1.5 y 16.0 $\mathrm{mg} / \mathrm{l}$, muestra una correlación lineal entre la frecuencia de fracturas óseas (en niños y adultos) y la severidad de la fluorosis dental. La prevalencia de fracturas óseas, en personas (13 a 60 años de edad) que consumieron durante nueve años agua con una $\left[\mathrm{F}^{-}\right]$en el rango de 1.5 a $8.5 \mathrm{mg} / \mathrm{l}$, fue de $30 \%$.

Las poblaciones de LAJ que consumen agua potable con una $\left[\mathrm{F}^{-}\right]$mayor de $4.0 \mathrm{mg} / \mathrm{l}$, sin lugar a duda están expuestas a enfermedades fluoróticas severas como son fluorosis esquelética, fracturas óseas, cáncer, trastornos gastrointestinales, y alteraciones renales. Las ciudades con mayores riesgos son: Encarnación de Díaz, Lagos de Moreno, Mexticacán, San Juan de los Lagos, Teocaltiche, Tepatitlán de Morelos y Valle de Guadalupe.

Los resultados de este estudio permiten establecer que los niños son los que están más expuestos a la acción tóxica del flúor, ya que los valores de ingestión total de $\mathrm{F}^{-} \mathrm{y}$ las dosis de exposición rebasan las recomendaciones sanitarias.

La mayoría de los habitantes de LAJ, incluyendo especialistas en salud, desconocen la problemática del flúor y, por lo tanto, no practican acciones simples que les permitirían reducir los riesgos. Es muy probable que las enfermedades fluoróticas sean mal diagnosticadas.

Para reducir los riesgos potenciales de salud de los habitantes de LAJ, por el consumo prolongado de altos niveles de flúor se recomienda: a) estricta vigilancia para que no se comercialice agua embotellada con exceso de $\mathrm{F}^{-}$, ni de sal fluorada que haga que el agua potable tenga mas de $0.7 \mathrm{mg} \mathrm{F}^{-} / \mathrm{l} ; \mathrm{b}$ ) realizar los estudios epidemiológicos cada cinco años como lo establece la normatividad; c) establecer un programa de comunicación de riesgos para informar a la población la problemática del flúor y las acciones necesarias para minimizar riesgos tales como reducir el tiempo de hervido del agua y los alimentos, así como también evitar el uso del agua de la llave para la preparación de fórmulas para niños menores de dos años de edad; $d$ ) que las autoridades sanitarias reconozcan la importancia de la erradicación de este problema de salud pública, y e) fomentar la comercialización de filtros caseros para eliminar fluoruros y otros elementos traza del agua potable.

\section{Agradecimientos}

Agradecemos a las siguientes instituciones el apoyo para la realización de este trabajo: Agencia de Protección Ambiental de Estados Unidos (US EPA), Autotransportes Mezcala (ATM), El Colegio de la Frontera Norte, A.C. (El COLEF) y Universidad de Texas en El Paso (UTEP).

\section{Referencias}

1. Instituto N acional de Estadística, Geografía e Informática. X II Censo General de Población y Vivienda 2000. A guascalientes, A guascalientes, México: IN EG I; 2001.

2. Hurtado R, Gardea-Torresdey J,Tiemann KJ. Fluoride occurrence in tap water at "Los Altos de Jalisco", in the central Mexico region. Proceedings of the 2000 Conference on Hazardous W aste Research: Environmental $C$ hanges and Solutions to Resource D evelopment, Production, and Use; 2000 mayo 23-25; D enver (CO), USA. Manhattan (KS): Erikson LE, Ranking MM, Kansas State University: 211-219. 3. Secretaría de Salud. N orma 0 ficial Mexicana: N O M 127-SSA 1-1994. Salud ambiental, agua para uso y consumo humano. Límites permisibles de calidad y tratamientos a que debe someterse el agua para su potabilización. México, D.F.: Diario 0 ficial de la Federación DVIII 13, 18 de enero; 1996: 41. 
4. $\mathrm{N}$ ational Research Council. Health effects of ingested fluoride. Report of the Subcommittee on Health Effects of Ingested Fluoride. W ashington, DC: $N$ ational A cademy Press; 1993.

5. Grimaldo M, Borja-A burto VH, Ramírez AL, Ponce M, Rosas M, DíazBarriga F. Endemic fluorosis in San Luis Potosi, Mexico: I. Identification of risk factors associated with human exposure to fluoride. Environ Res 1995;68:25-30.

6. Choubisa SL. Endemic fluorosis in southern Rajasthan, India. Fluoride 2001;34(1):61-70.

7. ChakmaT, Rao PV, Singh SB, Tiwary RS. Endemic genu valgum and other bone deformities in two villages of Mandla district in central India. Fluoride 2000;33(4):187-195

8.Alarcón-Herrera MT, Martín-D omínguez IR,Trejo-Vazquez R, Rodríguez-D ozal S.W ell water fluoride, dental fluorosis, and bone fractures in the GuadianaValley of Mexico. Fluoride 2001;34(2):139-149. 9.Y iamouyiannis J. Fluoride the aging factor: How to recognize and avoid the devastating effects of fluoride. $3^{\text {rd }}$ edition. D elaware, 0 hio: Health Action Press; 1993.

10.Trejo-Vázquez R, Bonilla-Petriciolet A. Exposición a fluoruros del agua potable en la ciudad de Aguascalientes, México. Pan Am J Public Health 2001;10(2):108-113.

11. Barrandey-0 rozco SE, C abello-A rreola MV, Magaña-Ramírez J, Rodríguez-D omínguez E. Sal fluorurada, riesgo o beneficio para la población de la ciudad de Chihuahua. Rev AD M 1994;51(2):80-89. 12. Grijalva-H aro MI, Barba-Leyva ME, Laborín-A lvarez A. Ingestión y excreción de fluoruros en niños de Hermosillo, Sonora, México. Salud Publica Mex 2001;43(2):127-134.

13. Díaz-Barriga F, N avarro-Q uezada A, G rijalva MI, Grimaldo M, LoyolaRodríguez JP, O rtiz MD. Endemic fluorosis in México. Fluoride 1997;30(4):233-239.
14. Loyola-Rodríguez JP, Pozos-Guillén AJ, Rueda-González AM, VázquezMoctezuma S, de la Paz-D omínguez G. Factores a riesgo de fluorosis dental en San Luis Potosí, México. Revista AD M 1996;53(6):295-300. 15. Loyola-Rodríguez JP, Pozos-G uillén AJ, Hernández-Guerrero JC. Bebidas embotelladas como fuentes adicionales de exposición a flúor. Salud Publica Mex 1998;40(5):438-441.

16. Díaz-Barriga F, Leyva R, Q uistián J, Loyola-Rodríguez JP, Pozos A, Grimaldo M. Endemic fluorosis in San Luis Potosi, Mexico. IV. Sources of fluoride exposure. Fluoride 1997;30(4):219-222.

17. U. S. Environmental Protection A gency. Methods for chemical analysis of water and wastes -Method 340.2 -Fluoride. Storet $\mathrm{N} 0$. : 00950, Environmental monitoring and support laboratory Cincinnati, 0 hio: - 0 ffice of research and development- U.S. EPA. 1991.

18. Hurtado R, Gardea-Torresdey J. Environmental evaluation of fluoride in drinking water at "Los Altos de Jalisco", in the central Mexico region. J Toxicol Environ Health A 2004;67(20-22):1741-1753.

19. Martínez-Salgado H,Tovar-Zamora E, C hávez-Villasana A, Armendáriz D M, Baz-Díaz-Lombardo G. Consumo familiar e individual de sal de mesa en el Estado de México. Salud Publica Mex 1993;35(6):630-636. 20. 0 rtiz D, C astro L,Turrubiartes F, Milan J, Díaz-Barriga F.Assessment of the exposure to fluoride from drinking water in Durango, Mexico, using a geographic information system. Fluoride 1998;31(4):183-187. 21. Institute of Medicine. Fluoride. En: D ietary reference intakes for calcium, phosphorus, magnesium, vitamin $D$, and fluoride.W ashington, DC: N ational A cademy Press; 1997:288-313.

22. C ommittee on Medical Aspects of Food and N utrition Policy. Fluoride. Dietary reference values for food energy and nutrients for the United Kingdom. Londres VR: Her Majesty's Stationery O ffice; 1994. 23.W orld Health O rganization. Fluorides - Environmental Health Criteria 227. Ginebra:W HO ;2002. 